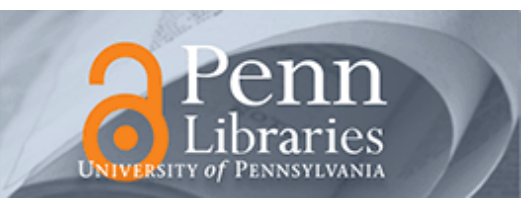

University of Pennsylvania

ScholarlyCommons

\title{
Charged Particle Distributions and Heat Transfer in a Discharge Between Geometrically Dissimilar Electrodes: From Breakdown to Steady State
}

Wei Qin

University of Pennsylvania, qinwei@mail.med.upenn.edu

Ira M. Cohen

University of Pennsylvania

Portonovo S. Ayyaswamy

University of Pennsylvania, ayya@seas.upenn.edu

Follow this and additional works at: https://repository.upenn.edu/meam_papers

Part of the Mechanical Engineering Commons

\section{Recommended Citation}

Qin, Wei; Cohen, Ira M.; and Ayyaswamy, Portonovo S., "Charged Particle Distributions and Heat Transfer in a Discharge Between Geometrically Dissimilar Electrodes: From Breakdown to Steady State" (2000). Departmental Papers (MEAM). 175.

https://repository.upenn.edu/meam_papers/175

Suggested Citation:

Qin, Wei, Ira M. Cohen and Portonovo S. Ayyaswamy. (2000). Charged particle distributions and heat transfer in discharge between geometrically dissimilar electrodes: From breakdown to a steady state. Physics of Plasmas. Vol. 7(2).

Copyright (2000) American Institute of Physics. This article may be downloaded for personal use only. Any other use requires prior permission of the author and the American Institute of Physics. The following article appeared in Physics of Plasmas and may be found at http://scitation.aip.org/getpdf/servlet/

GetPDFServlet?filetype=pdf\&id=PHPAEN000007000002000719000001\&idtype=cvips\&prog=normal\&doi=10.1063/ 1.873858

This paper is posted at ScholarlyCommons. https://repository.upenn.edu/meam_papers/175

For more information, please contact repository@pobox.upenn.edu. 


\title{
Charged Particle Distributions and Heat Transfer in a Discharge Between Geometrically Dissimilar Electrodes: From Breakdown to Steady State
}

\author{
Abstract \\ The low-current electric discharge from a fine wire anode to a planar cathode in atmospheric pressure air \\ is numerically simulated from high-voltage prebreakdown through electron temperature growth, then \\ ionization and consequent current growth to steady state, limited by a ballast resistor in the external \\ circuit. Conservation of number mass! for ions and electrons, Gauss' law for the self-consistent electric \\ field, and energy conservation for electrons have been solved from breakdown to steady state in a body \\ fitted coordinate system generated specifically for these two geometrically dissimilar electrodes. To \\ facilitate the discussion of the results, the discharge has been categorized under $\sim a$ ! electron \\ acceleration period, $\sim b$ ! charged particle generation period, $\sim c$ ! current increase and voltage drop period, \\ and $\sim$ d! current and voltage stabilization period. Results are given for transient electron, ion, and \\ temperature distributions in the gap as well as current growth and voltage drop across the gap. Heat flux \\ from the discharge to the wire is calculated. The numerical simulations were compared with experiments \\ performed under the same conditions on a wire bonding machine with very close correspondence.

\section{Disciplines} \\ Engineering | Mechanical Engineering

\section{Comments} \\ Suggested Citation: \\ Qin, Wei, Ira M. Cohen and Portonovo S. Ayyaswamy. (2000). Charged particle distributions and heat \\ transfer in discharge between geometrically dissimilar electrodes: From breakdown to a steady state. \\ Physics of Plasmas. Vol. 7(2). \\ Copyright (2000) American Institute of Physics. This article may be downloaded for personal use only. \\ Any other use requires prior permission of the author and the American Institute of Physics. The following \\ article appeared in Physics of Plasmas and may be found at http://scitation.aip.org/getpdf/servlet/ \\ GetPDFServlet?filetype=pdf\&id=PHPAEN000007000002000719000001\&idtype=cvips\&prog=normal\&doi=10.1063/ \\ 1.873858
}




\title{
Charged particle distributions and heat transfer in a discharge between geometrically dissimilar electrodes: From breakdown to steady state
}

\author{
Wei (Ivy) Qin, Ira M. Cohen, and P. S. Ayyaswamy \\ The University of Pennsylvania, Department of Mechanical Engineering and Applied Mechanics, \\ Philadelphia, Pennsylvania 19104
}

(Received 14 December 1998; accepted 18 October 1999)

\begin{abstract}
The low-current electric discharge from a fine wire anode to a planar cathode in atmospheric pressure air is numerically simulated from high-voltage prebreakdown through electron temperature growth, then ionization and consequent current growth to steady state, limited by a ballast resistor in the external circuit. Conservation of number (mass) for ions and electrons, Gauss' law for the self-consistent electric field, and energy conservation for electrons have been solved from breakdown to steady state in a body fitted coordinate system generated specifically for these two geometrically dissimilar electrodes. To facilitate the discussion of the results, the discharge has been categorized under (a) electron acceleration period, (b) charged particle generation period, (c) current increase and voltage drop period, and (d) current and voltage stabilization period. Results are given for transient electron, ion, and temperature distributions in the gap as well as current growth and voltage drop across the gap. Heat flux from the discharge to the wire is calculated. The numerical simulations were compared with experiments performed under the same conditions on a wire bonding machine with very close correspondence. (C) 2000 American Institute of Physics.
\end{abstract}

[S1070-664X(00)00902-2]

\section{INTRODUCTION}

When a high voltage is applied between a wire anode and a planar cathode, the few electrons present in the atmosphere are accelerated by the electric field and reach a very high energy in a short distance. When colliding with gas molecules, those electrons with sufficient energy (exceeding the ionization energy for the neutral particles) will ionize them and produce new electrons and ions. In the electric field, the positive ions and electrons move toward the negative and positive electrodes, respectively, and the current begins to flow in the gap. If the applied voltage is above a critical value, this avalanche of electrons produces sufficient charged particles so that the discharge becomes selfsustained and breakdown in the gap has occurred. A summary of numerical simulations of electric breakdown was given by Davis. ${ }^{1}$ Numerical simulations of the glow discharge between parallel plate electrodes were reported by Novak and Bartnikas, ${ }^{2}$ Pai, ${ }^{3}$ Pai and Guo, ${ }^{4}$ Meyyappan and Kreskovsky, ${ }^{5}$ and Graves and Jensen. ${ }^{6}$ In some of the works mentioned above, only the conservation equations for charged particles and Poisson's equation are solved. (See Refs. 2, 3, and 4) Others also calculated the energy conservation equations for the electrons. (See Refs. 5 and 6.)

Graves and Jensen, ${ }^{6}$ and Park and Economou ${ }^{7}$ used the following equation to calculate the ionization rate:

$$
P_{i}\left(N_{e}\right)=N_{e} k_{i o} \times \exp \left(-\frac{E_{i}}{k T_{e}}\right),
$$

where $P_{i}\left(N_{e}\right)$ is the impact ionization rate, $k_{i o}$ is called the pre-exponential coefficient or the ionization rate coefficient pre-exponential factor, $E_{i}$ is the ionization energy for the gas molecules, $N_{e}$ is the electron density, and $T_{e}$ is the electron temperature. From the above equation, the charged particle generation is proportional to electron density and dependent exponentially on electron temperature.

Heat transfer to the anode was given by Cobine and Burger $^{8}$ as $J_{e}\left(V_{a}+\phi+V_{T}\right)$, where $J_{e}$ is current density to the anode, $V_{a}$ is the anode drop, $\phi$ is the work function, and $V_{T}$ is the thermal energy carried by the electrons.

A more complicated geometry involving two geometrically dissimilar electrodes has been studied by Ramakrishna et al., ${ }^{9,10}$ Donovan and Cohen, ${ }^{11}$ Jog et al. ${ }^{12}$ for a wire-toplane discharge, and by Qin et al. ${ }^{13}$ for a wire-to-ring discharge. All of the studies cited above have assumed that the voltages prescribed at the electrodes remain fixed. The fixed voltage approximation is not realistic, since, as the current increases in the gap, the voltage between the gap drops because the electric circuit external to the discharge always has a ballast resistance. With the fixed voltage approximation, convergence difficulties seem to have been encountered during the solution procedure because current growth is unbounded as plasma conductivity increases with ionization. In the calculation presented here, the voltage between the two electrodes is adjusted after each time step according to the current flow in the gap. The simulation includes investigation of the discharge development and ionization growth from the initial electrostatic field between the electrodes.

All of the previously published studies related to wireto-plane discharges (Refs. 9-13) have exploited the advantages arising out of posing the problem in a special coordinate system. A prolate-spheroidal coordinate system has been employed for the wire-to-plane discharge, with the wire approximated by a hyperboloid of revolution. A major advantage with the use of this special coordinate system is that 
the initial potential distribution before the breakdown may be obtained analytically. Also, the wire electrode is a coordinate surface in a conventional orthogonal coordinate system. However, the result so obtained may be less accurate due to the approximation of the wire shape. The present formulation is based on a cylindrical anode, and is a more realistic and accurate approach. A body fitted coordinate system is used here. This also improves the accuracy of the simulation.

Another major difference between the work presented in this paper and the previous thesis researches by Ramakrishna, ${ }^{14}$ Donovan, ${ }^{15}$ and $\mathrm{Jog}^{16}$ is the implementation of the electron impact ionization term. In Ramakrishna's and Donovan's studies, the electron energy was not calculated. In Jog's study, the electron temperature was calculated. However, their ionization rate was not directly related to the electron temperature. They all used the following equation in their simulations where the ionization rate is dominated by electric field intensity:

$$
P_{i}\left(N_{e}\right)=A_{i} p \mu_{e} E e^{-B_{i} /(E p)},
$$

where constants $A_{i}$ and $B_{i}$ depend on the type of gas, $E$ is the electric field intensity, $p$ is the gas pressure, and $\mu_{e}$ is the electron mobility. In this paper, the electron temperature is calculated and the ionization rate is given by Eq. (1). This implementation has proven to be more accurate because it relates the ionization rate directly to the electron temperature. A comparison of the results between this and the previous work will be discussed later.

\section{BODY FITTED COORDINATE SYSTEMS}

A body fitted coordinate system will be used to solve the electronic breakdown problem and subsequent steady discharge between a thin gold wire $25.4 \mu \mathrm{m}$ in diameter and a flat plate beneath it. The gap length is set to be ten times the wire diameter. After the breakdown, the plasma heating process will start. The two advantages of using a body fitted coordinate system are that the boundary conditions become easier to implement, and they can be applied more accurately. For example, in a cylindrical coordinate system, the boundary consisting of the nascent ball surface (hemispherical wire tip) will have a zig-zag shape. In a body fitted coordinate system, the nascent ball surface can be represented exactly because the boundary will be considered as a constant coordinate line. The trade-off is an increase in the complexity of the transformed equations. Since we are modeling a problem with a complicated geometry, the use of a body fitted coordinate system will improve the accuracy. Once the code for generating the body fitted coordinate system is written, it is very easy to generate a different solution domain such as one with a different gap length or different wire diameter. The elliptic grid generation technique was used. This method creates a very smooth interior grid, even for nonsmooth boundary data. Grid smoothness is necessary to achieve low truncation error in the solution of the finite difference equations. Another advantage of the elliptic approach is that the interior grid is relatively insensitive to the boundary parametrization. A more detailed description of the body fitted coordinate system is given in Ref. 17 .
The purpose of the grid generation is to transform a physical domain $(x, y)$, or $(r, z)$ in this case to a unit square logic domain $(\xi, \eta)$. The boundaries of the physical domain will be transformed into $\xi=0, \xi=1, \eta=0$, and $\eta=1$, respectively. In the new coordinate system, the boundary lines will be coincident with the constant coordinate lines. Therefore, this kind of coordinate system is called a body fitted coordinate system.

In the discharge simulation, the solution domain can be considered to be bounded by four sides; the bottom side is the cathode surface, the top side is the wire anode surface, the left side is the axis of symmetry, the right side is the outer boundary. In order to map this physical domain to the logic domain $(\xi, \eta)$, the cathode surface is mapped into $\eta$ $=0,0 \leqslant \xi \leqslant 1$, the wire surface is mapped into $\eta=1,0 \leqslant \xi$ $\leqslant 1$, the axis of symmetry is mapped into $\xi=0,0 \leqslant \eta \leqslant 1$, and the outer surface is mapped into $\xi=1,0 \leqslant \eta \leqslant 1$. The final curvilinear coordinate system for the wire to plane domain is governed by the following elliptic equations:

$$
\begin{gathered}
a_{11} \frac{\partial^{2} r}{\partial \xi^{2}}-2 a_{12} \frac{\partial^{2} r}{\partial \xi \partial \eta}+a_{22} \frac{\partial^{2} r}{\partial \eta^{2}}=0, \\
a_{11} \frac{\partial^{2} z}{\partial \xi^{2}}-2 a_{12} \frac{\partial^{2} z}{\partial \xi \partial \eta}+a_{22} \frac{\partial^{2} z}{\partial \eta^{2}} \\
=-Q_{0} \operatorname{sgn}\left(\eta-\eta_{0}\right) e^{-\left|\eta-\eta_{0}\right|}
\end{gathered}
$$

where

$$
a_{11}=r_{\eta}^{2}+z_{\eta}^{2}, \quad a_{22}=r_{\xi}^{2}+z_{\xi}^{2}, \quad a_{12}=r_{\xi} r_{\eta}+z_{\eta} z_{\xi},
$$

and $Q_{0}$ is the attraction factor. If $Q_{0}>0$, lines are attracted to $\eta=\eta_{0}$, while if $Q_{0}<0$, lines are repelled. In the simulation, $Q_{0}=6, \eta_{0}=0.99$ was used. Therefore, the grid lines are concentrated near the wire surface, which is the coordinate line $\eta=1$. A similar control parameter can be used in the $r$ direction if needed. In this program, it is not implemented, since the current setup is sufficient. On the wire and plane surfaces, the Dirichlet boundary conditions are used. For the left and right boundaries, the Neumann boundary conditions are used. The boundary conditions are given below:

On planar cathode $(\eta=0), \quad r=r_{t}(\xi), \quad z=0 ;$

on wire anode $(\eta=1), \quad r=r_{b}(\xi), \quad z=z_{b}(\xi)$;

on the axis of symmetry $(\xi=0), \quad r=0$,

$\partial z / \partial \xi=0$

on the outside boundary $(\xi=1), \quad \partial \mathbf{n} / \partial \xi=0$,

$r^{2}+z^{2}=R_{\text {domain }}^{2}$

The grid distributions on the top and bottom boundaries are $r_{t}, r_{b}, z_{b}$ and are predetermined, $\mathbf{n}$ is the unit vector from the outer surface, $R_{\text {domain }}$ is the radius of the solution domain; it is taken to be 40 times the wire diameter in the simulation. Figure 1 shows a schematic of the discharge and circuit. The above equations with the boundary conditions are solved to determine $(r, z)$ as a function of $\xi, \eta$ using second-order central differences. The approximation of the second derivatives will involve nine neighboring 


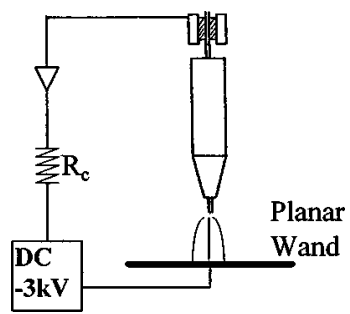

FIG. 1. Schematic of the discharge and circuit with the planar cathode.

points. A successive over-relaxation (SOR) method is used to solve the equation. The over-relaxation coefficient $\omega$ is equal to 1.6.

A plot of the entire solution domain (a quarter-circle) is shown in Fig. 2. The thin gold wire is approximated by a cylinder with a semispherical tip.

\section{INITIAL DISTRIBUTION OF ELECTRIC FIELD}

In the present study, as a first step, the electric field distribution established between the wire and plane in a neutral gas prior to breakdown is calculated. The Laplace equation governing the initial potential distribution in a neutral gas is given below with the boundary conditions. The governing equation is

$\nabla \cdot \nabla \Psi=0$

with the following boundary conditions:

On wire electrode, $\Psi=0$;

on planar electrode, $\quad \Psi=\Psi_{c}$;

on the outside boundary, $\partial \Psi / \partial n=0$.

Here $\Psi$ is the potential of the electric field. The potential of the gold wire is zero since the wire is grounded. $\Psi_{c}$ is the voltage of the planar cathode, which is initially taken to be a negative constant.

Laplace's equation governing the potential distribution can be derived for the body fitted coordinate system. With the body fitted coordinate system $(\xi, \eta)$,

$r=r(\xi, \eta), z=z(\xi, \eta)$.

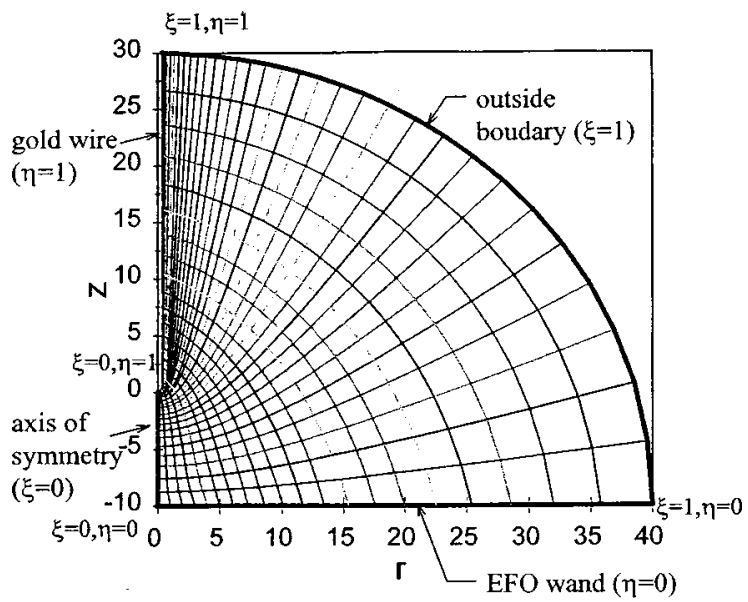

FIG. 2. Body fitted coordinate system for the arc discharge.
The Jacobian of this transformation is

$$
J=r_{\xi} z_{\eta}-r_{\eta} z_{\xi}
$$

The derivatives with respect to $r$ and $z$ are

$$
\begin{aligned}
& \psi_{r}=\frac{1}{J}\left(\psi \psi_{\xi} z_{\eta}-\psi \eta_{\eta} z_{\xi}\right)=\frac{1}{J}\left[\left(\psi z_{\eta}\right)_{\xi}-\left(\psi z_{\xi}\right)_{\eta}\right], \\
& \psi_{z}=\frac{1}{J}\left(\psi \psi_{\eta} r_{\xi}-\psi_{\xi} r_{\eta}\right)=\frac{1}{J}\left[\left(\psi r_{\xi}\right)_{\eta}-\left(\psi r_{\eta}\right)_{\xi}\right] .
\end{aligned}
$$

Here, $\psi=\Psi / \Psi_{R}$. The second form of the above equations is called the conservative or symmetric form of the transformed derivatives. Using both forms and rewriting Eq. (2),

$$
\frac{\partial}{\partial r}\left(r \frac{\partial \psi}{\partial r}\right)+\frac{\partial}{\partial z}\left(r \frac{\partial \psi}{\partial z}\right)=0
$$

Using Eqs. (3) and (4), Laplace's equation in the body fitted coordinate system becomes

$$
\frac{\partial}{\partial \xi}\left(\alpha \frac{\partial \psi}{\partial \xi}\right)-\frac{\partial}{\partial \xi}\left(\beta \frac{\partial \psi}{\partial \eta}\right)-\frac{\partial}{\partial \eta}\left(\beta \frac{\partial \psi}{\partial \xi}\right)+\frac{\partial}{\partial \eta}\left(\gamma \frac{\partial \psi}{\partial \eta}\right)=0,
$$

where

$$
\begin{aligned}
& \alpha=\frac{r}{J}\left(r_{\eta}^{2}+z_{\eta}^{2}\right), \quad \beta=\frac{r}{J}\left(r_{\xi} r_{\eta}+z_{\xi} z_{\eta}\right), \\
& \gamma=\frac{r}{J}\left(r_{\xi}^{2}+z_{\xi}^{2}\right) .
\end{aligned}
$$

Here, $\alpha, \beta$, and $\gamma$ are functions of the new coordinate system. Their values can be obtained once the coordinate system is established. The boundary conditions are very simple due to the use of a body fitted coordinate system:

$$
\begin{aligned}
& \psi(\xi, 0)=\psi_{c}, \quad \psi(\xi, 1)=0, \quad \frac{\partial \psi}{\partial \xi}(0, \eta)=0, \\
& \frac{\partial \psi}{\partial \xi}(1, \eta)=0 .
\end{aligned}
$$

Equation (5) is solved by a control volume method with body fitted coordinates. The wire diameter is taken to be 25.4 $\mu \mathrm{m}$. The initial potential distributions are given in Fig. 3, where the wire surface is an equipotential line of $0 \mathrm{~V}$ and the cathode surface is an equipotential line of $-3000 \mathrm{~V}$. The equipotential lines are concentrated around the wire tip, so the electric field is much stronger near the wire than close to the cathode. Hence, the increase of electron temperature and the ionization of gas particles are expected to commence around the wire tip.

The result obtained above is used as an initial condition for the transient breakdown problem.

\section{TRANSFORMATION OF THE CONSERVATION EQUATIONS INTO BODY FITTED COORDINATE SYSTEM}

In this model, the pressure is high enough (atmospheric pressure) so that the electron mean-free path is the smallest 


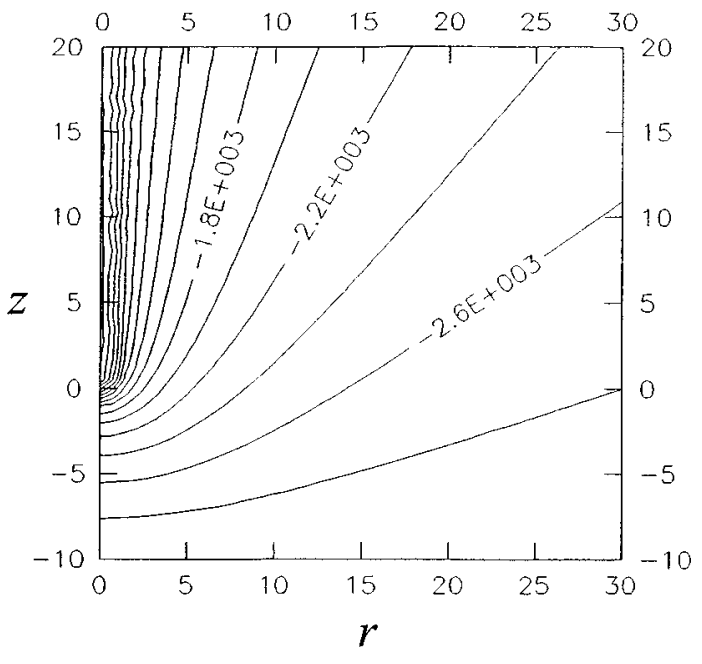

FIG. 3. Equipotential lines for the wire to plane configuration. Equipotential lines every $200 \mathrm{~V}$. Here $r$ and $z$ are lengths in units of wire diameters (25.4 $\mu \mathrm{m})$.

characteristic length. This allows us to use the continuum model to describe the electron and ion behavior. We now consider a collision-dominated, low-energy, two-temperature plasma consisting of three species-electrons, positive ions, and neutrals. We assume that each of the species is in equilibrium with its like particle, and the background gas is homogeneous and at rest. The ions exchange energy efficiently with neutrals and are considered to be at the gas temperature. In contrast, the electrons do not lose much energy during elastic collisions with neutrals and the temperature of the electrons is calculated from the electron energy equation. We use the formulation for a weakly ionized plasma derived by Wilkins and Gyftopoulos. The equations presented below are given in Refs. 18 and 19. We assume the following conditions and neglect thermal diffusion.

(a) The mass of the negative charge carriers is much smaller than that of the positive charge carriers and neutrals.

(b) The neutral-ion collision frequency is much smaller than the neutral-neutral collision frequency. This leads to the conclusion that the neutral distribution function is in its equilibrium form.

(c) Any characteristic mean-free path is much smaller than the characteristic length of the plasma. A continuum approach can be used to derive the hydrodynamic equations.

(d) The drift velocities are much smaller than the random velocities. The mass average velocity is zero.

The mass conservation equations are

$$
\frac{\partial N_{e, i}}{\partial t}+\boldsymbol{\nabla} \cdot \mathbf{j}_{e, i}=P_{i}\left(N_{e}\right),
$$

where the electron and the ion fluxes are given by

$$
\begin{aligned}
& \mathbf{j}_{e, i}=-\frac{\mu_{e, i}}{e} \nabla p_{e, i} \pm \mu_{e, i} N_{e, i} \nabla \Psi, \\
& p_{e, i}=N_{e, i} k T_{e, i} .
\end{aligned}
$$

The energy conservation equation for electrons is represented by

$$
\frac{\partial}{\partial t}\left(\frac{3}{2} N_{e} k T_{e}\right)+\nabla \cdot \mathbf{Q}_{e}=-e \mathbf{j}_{e} \cdot \mathbf{E}-Q_{\mathrm{in}},
$$

where the electron heat flux is given by

$$
\mathbf{Q}_{e}=\frac{5}{2} k T_{e} \mathbf{j}_{e}-\kappa_{e} \boldsymbol{\nabla} T_{e} .
$$

$Q_{\text {in }}$ is the loss of energy of electrons due to inelastic collisions:

$$
Q_{\mathrm{in}}=e \Psi_{i} P_{i}\left(N_{e}\right) .
$$

Equation (10) assumes that the only inelastic collisions are ionization collisions. Although we cannot justify this assumption here, our later results show that the numerical simulations are very close to the experimental measurements, lending validity to this model.

The self-consistent electric field is given by Gauss' law

$$
\boldsymbol{\nabla} \cdot \mathbf{E}=\frac{e}{\epsilon_{0}}\left(N_{i}-N_{e}\right),
$$

where

$$
\mathbf{E}=-\nabla \Psi .
$$

Here subscript $e$ refers to electrons and $i$ refers to ions, $p$ is pressure, $N$ is charged particle number density, $\mathbf{E}$ is electric field intensity, $T$ is temperature, and $\mu$ is the mobility of charged species. The value of $\mu$ for air can be found in Refs. 20 and 21 . Here $\kappa_{e}$ is the thermal conductivity of electrons, which is a function of $N_{e}$ and $T_{e}$ (see Ref. 22), and is taken here to be

$$
\kappa_{e}=1.14 \times 10^{-28} N_{e} T_{e}(\mathrm{~W} / \mathrm{mK}) ;
$$

$\Psi_{i}$ is the ionization potential, $k$ is the Boltzmann constant, $\epsilon_{0}$ is permittivity of free space, and $e$ is magnitude of electric charge on a single electron.

The ionization rate by electron impact, $P_{i}\left(N_{e}\right)$, is considered to be dependent on the electron temperature and is given by Eq. (1), written as

$$
P_{i}\left(N_{e}\right)=N_{e} k_{i o} \times \exp \left(-\frac{e \Psi_{i}}{k T_{e}}\right) .
$$

The ionization rate coefficient $k_{i o}$ is (see Ref. 5):

$$
k_{i o}=N_{n} \sigma_{T}\left(\frac{8 k T_{e}}{\pi m_{e}}\right)^{1 / 2}\left(1+\frac{e \Psi_{i}}{k T_{e}}\right),
$$

where $N_{n}$ is the bulk gas density, and $\sigma_{T}$ is the collision cross section. Recombination has been neglected because we have shown by direct computation that its effect is negligible $\left(<10^{-4}\right)$ during the time scale of interest.

We introduce the following normalizations to obtain a nondimensional formulation. Length is normalized by wire diameter $d$, which is taken to be $25.4 \mu \mathrm{m}$. The temperatures are normalized by the ambient temperature $T_{\infty}$. The number densities are normalized by the number density value which results in a Debye length equal to the wire diameter (d), $N_{R}=k T_{\infty} \epsilon_{0} /\left(e^{2} d^{2}\right)$. The potential and the electric field are normalized by $\Psi_{R}=k T_{\infty} / e$ and $E_{R}=\Psi_{R} / d$, respectively. 
The time is normalized by the breakdown time scale $t_{R}$ $=d /\left(\mu_{e} E_{R}\right)$, which is equal to the reference length $d$ divided by the electron drift velocity of $\mu_{e} E_{R}$.

In the body fitted coordinates, we have Poisson's equation as

$$
\begin{aligned}
& \frac{\partial}{\partial \xi}\left(\alpha \frac{\partial \psi}{\partial \xi}\right)-\frac{\partial}{\partial \xi}\left(\beta \frac{\partial \psi}{\partial \eta}\right)-\frac{\partial}{\partial \eta}\left(\beta \frac{\partial \psi}{\partial \xi}\right)+\frac{\partial}{\partial \eta}\left(\gamma \frac{\partial \psi}{\partial \eta}\right) \\
& =r J\left(n_{e}-n_{i}\right) .
\end{aligned}
$$

The mass conservation equations can be written as

$$
\begin{aligned}
\frac{\partial n_{e}}{\partial t} & -\frac{1}{r J} \frac{\partial}{\partial \xi}\left[\alpha\left(\frac{\partial\left(T_{e} n_{e}\right)}{\partial \xi}-n_{e} \frac{\partial \psi}{\partial \xi}\right)\right]+\frac{1}{r J} \frac{\partial}{\partial \xi}\left[\beta \left(\frac{\partial\left(T_{e} n_{e}\right)}{\partial \eta}\right.\right. \\
& \left.\left.-n_{e} \frac{\partial \psi}{\partial \eta}\right)\right]+\frac{1}{r J} \frac{\partial}{\partial \eta}\left[\beta\left(\frac{\partial\left(T_{e} n_{e}\right)}{\partial \xi}-n_{e} \frac{\partial \psi}{\partial \xi}\right)\right] \\
& -\frac{1}{r J} \frac{\partial}{\partial \eta}\left[\gamma\left(\frac{\partial\left(T_{e} n_{e}\right)}{\partial \eta}-n_{e} \frac{\partial \psi}{\partial \eta}\right)\right]=g_{m},
\end{aligned}
$$

$$
\begin{gathered}
\frac{\partial n_{i}}{\partial t}-\frac{F_{i}}{r J} \frac{\partial}{\partial \xi}\left[\alpha\left(\frac{\partial\left(T_{i} n_{i}\right)}{\partial \xi}+n_{i} \frac{\partial \psi}{\partial \xi}\right)\right]+\frac{F_{i}}{r J} \frac{\partial}{\partial \xi}\left[\beta \left(\frac{\partial\left(T_{i} n_{i}\right)}{\partial \eta}\right.\right. \\
\left.\left.\left.+n_{i} \frac{\partial \psi}{\partial \eta}\right)\right]+\frac{F_{i}}{r J} \frac{\partial}{\partial \eta}\left[\beta\left(\frac{\partial\left(T_{i} n_{i}\right)}{\partial \xi}+n_{i} \frac{\partial \psi}{\partial \xi}\right)\right]\right] \\
-\frac{F_{i}}{r J} \frac{\partial}{\partial \eta}\left[\gamma\left(\frac{\partial\left(T_{i} n_{i}\right)}{\partial \eta}+n_{i} \frac{\partial \psi}{\partial \eta}\right)\right]=g_{m},
\end{gathered}
$$

where the source term is

$$
g_{m}=F_{s} n_{e} T_{e}^{1 / 2}\left(1+\psi_{i} / T_{e}\right) \exp \left(-\psi_{i} / T_{e}\right) .
$$

The energy conservation equation can be written as

$$
\begin{gathered}
\left.\frac{\partial n_{e} T_{e}}{\partial t}-\frac{5}{3 r J} \frac{\partial}{\partial \xi}\left[\alpha T_{e}\left(\frac{\partial\left(T_{e} n_{e}\right)}{\partial \xi}-n_{e} \frac{\partial \psi}{\partial \xi}\right)\right]+\frac{5}{3 r J} \frac{\partial}{\partial \xi}\left[\beta T_{e}\left(\frac{\partial\left(T_{e} n_{e}\right)}{\partial \eta}-n_{e} \frac{\partial \psi}{\partial \eta}\right)\right]+\frac{5}{3 r J} \frac{\partial}{\partial \eta}\left[\beta T_{e}\left(\frac{\partial\left(T_{e} n_{e}\right)}{\partial \xi}-n_{e} \frac{\partial \psi}{\partial \xi}\right)\right]\right] \\
-\frac{5}{3 r J} \frac{\partial}{\partial \eta}\left[\gamma T_{e}\left(\frac{\partial\left(T_{e} n_{e}\right)}{\partial \eta}-n_{e} \frac{\partial \psi}{\partial \eta}\right)\right]-\frac{2 F_{c}}{3 r J}\left[\frac{\partial}{\partial \xi}\left(\alpha \kappa_{e} \frac{\partial T_{e}}{\partial \xi}\right)-\frac{\partial}{\partial \xi}\left(\beta \kappa_{e} \frac{\partial T_{e}}{\partial \eta}\right)-\frac{\partial}{\partial \eta}\left(\beta \kappa_{e} \frac{\partial T_{e}}{\partial \xi}\right)+\frac{\partial}{\partial \eta}\left(\gamma \kappa_{e} \frac{\partial T_{e}}{\partial \eta}\right)\right] \\
=-\frac{2}{3 r J}\left(\alpha \frac{\partial n_{e} T_{e}}{\partial \xi} \frac{\partial \psi}{\partial \xi}-\beta \frac{\partial n_{e} T_{e}}{\partial \xi} \frac{\partial \psi}{\partial \eta}-\beta \frac{\partial n_{e} T_{e}}{\partial \eta} \frac{\partial \psi}{\partial \xi}+\gamma \frac{\partial n_{e} T_{e}}{\partial \eta} \frac{\partial \psi}{\partial \eta}\right)+\frac{2 n_{e}}{3 r J}\left[\alpha\left(\frac{\partial \psi}{\partial \xi}\right)^{2}-2 \beta \frac{\partial \psi}{\partial \xi} \frac{\partial \psi}{\partial \eta}+\gamma\left(\frac{\partial \psi}{\partial \eta}\right)\right]+g_{e},
\end{gathered}
$$

where the loss of electron energy due to inelastic collisions is

$$
g_{e}=-\frac{2}{3} \psi_{i} F_{s} n_{e} T_{e}^{1 / 2}\left(1+\psi_{i} / T_{e}\right) \exp \left(-\psi_{i} / T_{e}\right) .
$$

In the above equations $n_{e, i}=N_{e, i} / N_{R}, \quad \psi=\Psi / \Psi_{R}, \quad F_{i}$ $=\mu_{i} / \mu_{e}, \quad F_{c}=t_{R} /\left(N_{R} d^{2} k\right), \quad F_{s}=t_{R} N_{n} \sigma_{T}\left(8 k T_{\infty} / \pi m_{e}\right)^{1 / 2}$. The boundary conditions are the following:

On plane electrode, $\quad \psi=\psi_{w}-R_{c} I_{w} / \Psi_{R}$,

$n_{e, i}(\xi, 0)=n_{0}, \quad T_{e}(\xi, 0)=1 ;$

on wire electrode, $\psi(\xi, 1)=0$,

$n_{e, i}(\xi, 1)=n_{0}, \quad T_{e}(\xi, 1)=T_{\text {wire }} ;$

on axis of symmetry, $\partial \psi / \partial \xi(0, \eta)=0$,

$\partial n_{e} / \partial \xi(0, \eta)=0, \quad \partial T_{e} / \partial \xi(0, \eta)=0 ;$

on outer boundary, $\partial \psi / \partial \xi(1, \eta)=0, \quad n_{e, i}(1, \eta)=n_{0}$,

$\partial T_{e} / \partial \xi(1, \eta)=0$

initial conditions are $n_{e, i}=n_{0}, \quad T_{e}=1$.

The wire and plane electrodes are assumed to have a charged particle density of $N_{0}$, which is the ambient density due to background radiation. The main reason to have the nonzero boundary conditions is to avoid a singularity at the boundaries. Because the magnitude of $N_{0}$ is very small, this assumption does not have a significant effect on the solution. $I_{w}$ is the current flow to the wire electrode and is given by

$$
I_{w}=\int e \cdot\left(\mathbf{j}_{i}-\mathbf{j}_{e}\right) \cdot d A_{w}
$$

In the above, $R_{c}$ is the outer circuit resistance, subscript $w$ refers to the wire, and $n_{0}$ is the dimensionless initial charged particle density. The parameters used in the calculation are given in Table I.

We note that the transport coefficients for the charged particles are functions of temperature. The system of equations is highly nonlinear, and the equations have to be solved simultaneously. A finite difference technique was employed to discretize the governing equations [see Eqs. (13)-(17)]. The grid size near the wire is set to be $0.002 d$ and is much smaller than the Debye length. The algebraic equations resulting from the discretization of the governing equations were solved using an alternating-direction implicit (ADI) scheme. $^{23}$ The diffusion and convection terms in the conservation equations were discretized by a power law method. ${ }^{24}$ At each time step, electric potential at the wire surface served as a boundary condition for the calculation in the plasma region. After each time step in the computation, relevant quantities of the plasma region and potential of the 


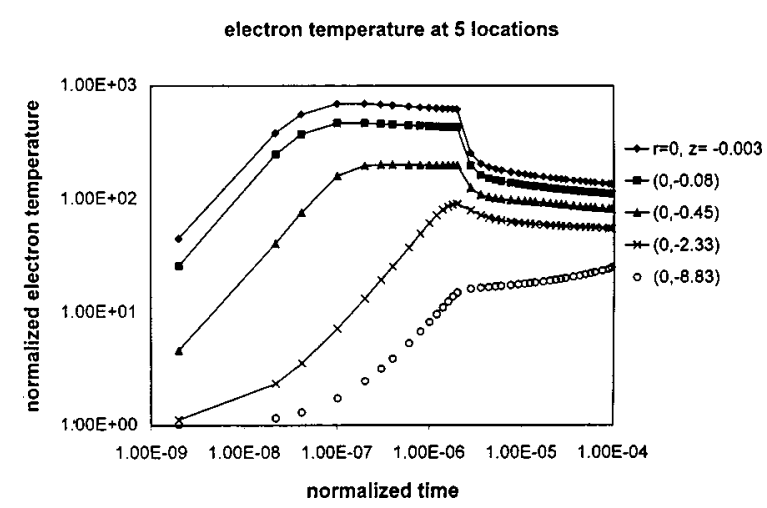

FIG. 4. Temperature development at five different locations along the axis of symmetry. Here $R_{c}=1 \times 10^{5} \Omega$, wire diameter $=25.4 \mu \mathrm{m}$, interelectrode gap $=0.254 \mathrm{~mm}$.

wire were updated. Equation (17) was used to update the potential boundary condition. During the development of the discharge, the temperature of the wire was considered to be unchanged. Since the temperature varies on a much longer time scale than the time scale of the breakdown, this is a reasonable assumption.

The heat transfer to the wire is estimated by taking into account (see Ref. 8) (a) electron enthalpy transport, (b) energy associated with electron absorption at the anode (wire) surface, and (c) conduction by electrons. These are expressed by

$$
q^{\prime \prime}=e \mathbf{j}_{e, n} \cdot\left(\frac{5}{2} \frac{k T_{e}}{e}+a_{e} \phi\right)-\kappa_{e}\left(\nabla T_{e}\right)_{, n},
$$

where $q^{\prime \prime}$ is the heat flux to the wire surface, $\mathbf{j}_{e, n}$ is the normal component of the electron density flux, $a_{e}$ is the accommodation coefficient $(=0.9)$, and $\phi$ is the work function $(=5 \mathrm{~V})$. The total heat input to the wire can be obtained by integrating the heat flux along the wire surface:

$$
q=\int q^{\prime \prime} d A_{w} .
$$

The calculations were carried out on an IBM RISC6000 workstation and a Sun workstation. The average run time for each simulation is around $5 \mathrm{~h}$. The results are discussed below.

TABLE I. Parameters used in the simulation.

$$
\begin{aligned}
& d=2.54 \times 10^{-5} \mathrm{~m} \\
& N_{0}=6 \times 10^{8} \mathrm{~m}^{-3} \\
& N_{n}=2.44 \times 10^{25} \mathrm{~m}^{-3} \\
& N_{R}=2.21 \times 10^{15} \mathrm{~m}^{-3} \\
& \Psi_{R}=0.026 \mathrm{~V} \\
& E_{R}=1.02 \times 10^{3} \mathrm{~V} / \mathrm{m} \\
& p=1.01 \times 10^{5} \mathrm{~Pa} \\
& T_{\infty}=300 \mathrm{~K} \\
& T_{i}=300 \mathrm{~K} \\
& t_{R}=0.717 \times 10^{-6} \mathrm{~s} \\
& \Psi_{i}=14.87 \mathrm{eV}^{-19} \mathrm{~m}^{2} \\
& \sigma_{T}=5 \times 10^{-19} \\
& \mu_{e}=0.0348 \mathrm{~m}^{2} / \mathrm{V} \mathrm{s} \\
& \mu_{i}=1.7 \times 10^{-4} \mathrm{~m}^{2} / \mathrm{V} \mathrm{s}
\end{aligned}
$$

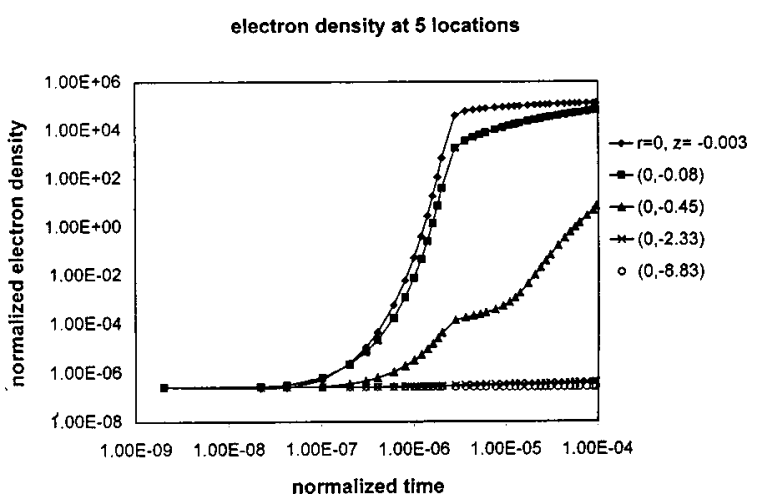

FIG. 5. Electron density development at five different locations. Here $R_{c}$ $=1 \times 10^{5} \Omega$, wire diameter $=25.4 \mu \mathrm{m}$, interelectrode $\mathrm{gap}=0.254 \mathrm{~mm}$.

\section{RESULTS AND DISCUSSION}

First, the timewise development of charged particle densities, electron temperature, current, and heat flux to the wire are analyzed. The spatial distributions of these variables are then considered. The influence of several key parameters on the results is also studied. These key parameters include the ballast resistor in the outer circuit, the length of the gap, and the wire diameter. Finally, the numerical results are compared to the results from experiments for potential, current change, and heat input to the wire.

\section{A. Time development of charged particle densities, electron temperature, potential across the gap and current to the wire}

The normalization of Eqs. (13) -(17) enables us to examine the short time behavior of the prebreakdown stage. The growth of electron temperature is given in Fig. 4. The electron and ion densities are given in Figs. 5-6. The electric field potential and the current flow in the gap are given in Fig. 7. The total heat input to the wire is given in Fig. 8. In these graphs, time, densities, and temperature are all normalized by reference quantities given in Table I. The reference time is about $10^{-6} \mathrm{~s}$ and the reference density is about $10^{-10}$ of the neutral particle density, the reference temperature is the ambient temperature, which is $300 \mathrm{~K}$. Figures 4-7 show the development of the electron temperature at five different

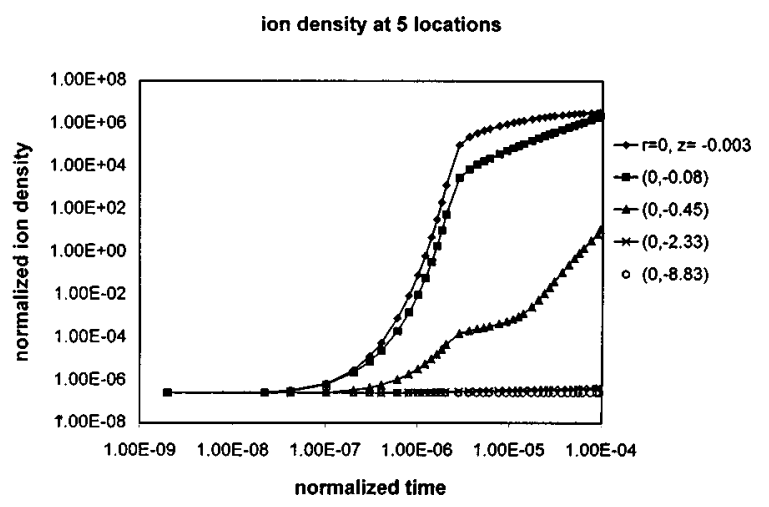

FIG. 6. Ion density development at five different locations. Here $R_{c}=1$ $\times 10^{5} \Omega$, wire diameter $=25.4 \mu \mathrm{m}$, interelectrode gap $=0.254 \mathrm{~mm}$. 


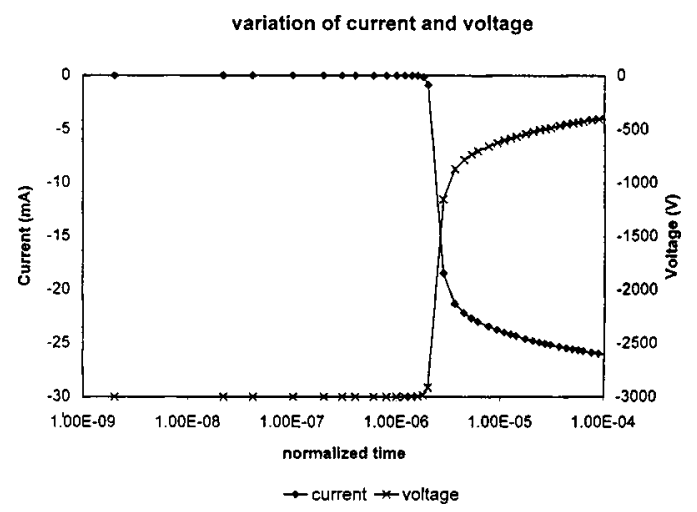

FIG. 7. Potential drop and current growth through time. Here $R_{c}=1$ $\times 10^{5} \Omega$, wire diameter $=25.4 \mu \mathrm{m}$, interelectrode gap $=0.254 \mathrm{~mm}$.

locations along the axis of symmetry. Here $(0,0)$ represents the tip of the wire, $(0,-10)$ represents the center of the wand.

By examining the results, the discharge can be categorized into four stages, as indicated below.

\section{Stage 1. electron acceleration period (normalized time $<10^{-7}$ )}

A normalized time of $10^{-7}$ corresponds to actual time of $10^{-13} \mathrm{~s}$ using a reference time of $t_{R}=0.717 \times 10^{-6} \mathrm{~s}$. In this period, the electron kinetic energy increases dramatically due to acceleration in the electric field, while the electron and ion densities remain the same. The collision time for electrons is around $10^{-13} \mathrm{~s}$, which can be estimated by calculating the electron collision frequency $\nu_{e n}$,

$$
\begin{aligned}
\nu_{e n} \approx e /\left(m_{e} \mu_{e}\right) & \approx 1.6 \times 10^{-19} /\left(9.1 \times 10^{-31} \times 0.0348\right) \\
& \approx 0.5 \times 10^{13}\left(\mathrm{~s}^{-1}\right) .
\end{aligned}
$$

Initially, the electrons are at a uniform temperature. Under the electric field, the electrons begin to accelerate and reach a higher kinetic energy. We can see that the temperature increases first near the wire tip where the electric field is strongest (Fig. 4), since the electric energy dissipation is proportional to the square of electric field intensity. The temperature is also higher near the wire tip than away from the

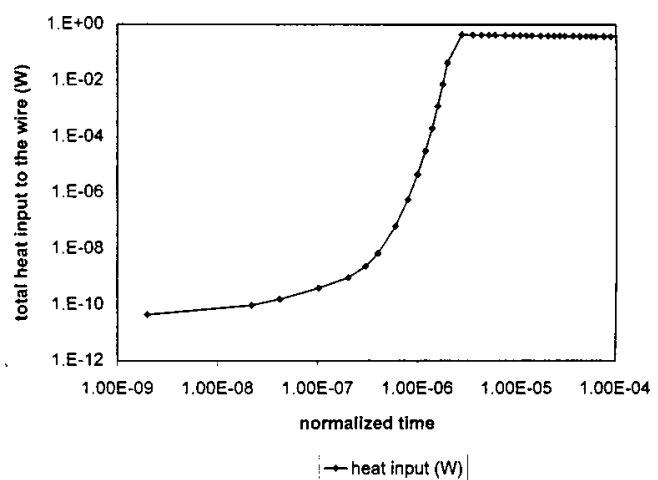

FIG. 8. Total heat input to the wire as a function of time. Here $R_{c}=1$ $\times 10^{5} \Omega$, wire diameter $=25.4 \mu \mathrm{m}$, interelectrode gap $=0.254 \mathrm{~mm}$.

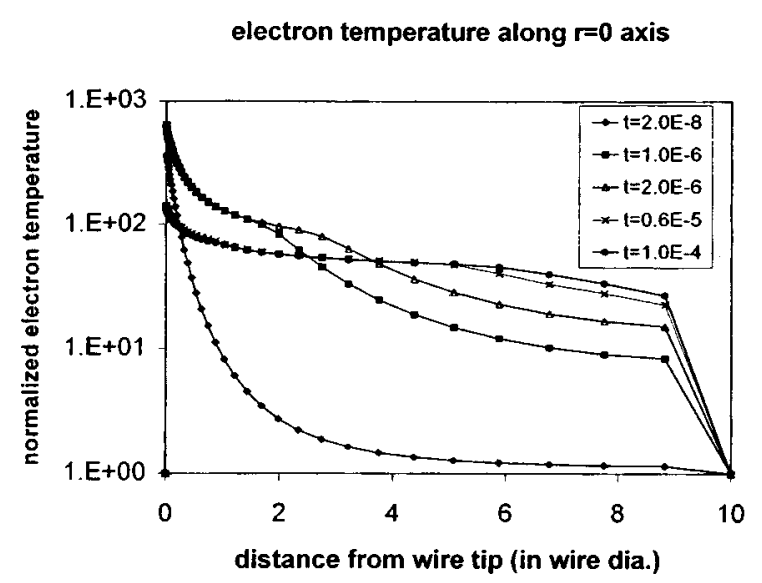

FIG. 9. Electron temperature distribution along the axis of symmetry. Here $R_{c}=1 \times 10^{5} \Omega$, wire diameter $=25.4 \mu \mathrm{m}$, interelectrode $\mathrm{gap}=0.254 \mathrm{~mm}$.

wire tip at any instant. In the meantime, the charged particle densities remain unchanged since inelastic collisions have not yet occurred at this time (Figs. 5 and 6).

\section{Stage 2: charged particle generation period $\left(10^{-7}<\right.$ normalized time $\left.<10^{-6}\right)$}

In this period, the electron and ion densities increase while the electron temperature near the wire changes very little. The temperature away from the wire keeps on increasing until the ionization rate reaches a high level. At a certain instant, the temperature can be more than $10^{5} \mathrm{~K}$ (more than $10 \mathrm{eV}$ ) near the wire tip, which is around the same magnitude as the ionization potential $(14.87 \mathrm{eV})$. The charged particle densities start to increase once the inelastic collisions start.

Figures 5 and 6 show the development of charged particle densities at five different locations along the axis of symmetry. The charged particle densities first rise near the wire tip, where the electron energy first approaches the ionization energy of the neutral gas. The increase of charged particle densities begins much later than the electron temperature increase, since only the high-energy electrons can ionize the neutral particles. The correlation of the tempera-

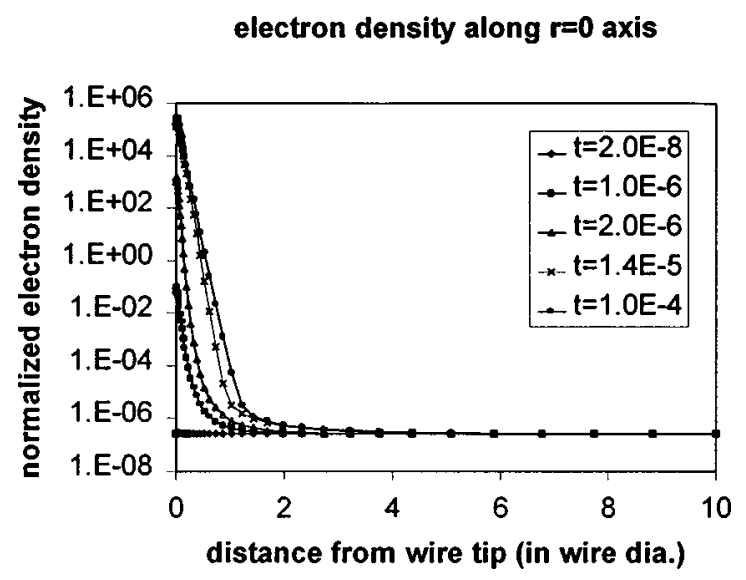

FIG. 10. Electron density distribution along the axis of symmetry. Here $R_{c}=1 \times 10^{5} \Omega$, wire diameter $=25.4 \mu \mathrm{m}$, interelectrode gap $=0.254 \mathrm{~mm}$. 
ion density along $r=0$ axis

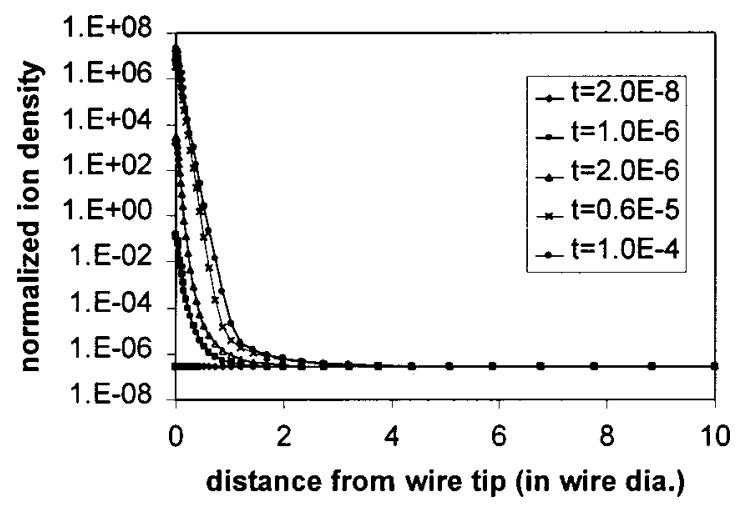

FIG. 11. Ion density distribution along the axis $R_{c}=1 \times 10^{5} \Omega$, wire diameter $=25.4 \mu \mathrm{m}$, interelectrode gap $=0.254 \mathrm{~mm}$.

ture drop and the density increase can be seen by comparing the temperature and density plots. Figures $4-6$ show that the sharp increase of the charged particle densities corresponds to the flattening of the electron temperature. As the energy of electrons is lost in inelastic collisions with the neutral particles, the electron temperature stops growing.

\section{Stage 3. current increase and voltage drop period $\left(10^{-6}<\right.$ normalized time $\left.<10^{-5}\right)$}

In this period, the current starts to flow to the electrodes while the potential drops (Fig. 7). The heat flux also increases with the current (Fig. 8).

The current in the gap is very small for short times. As the charged particle densities increase, the current begins to increase at a nondimensional time of about $10^{-6}$, as shown in Fig. 7. At the same time, the voltage between the gap begins to drop, as shown in Fig. 7. As the electric field intensity drops, the temperature of the electrons decreases and the charged particle densities saturate until a steady state is reached.

\section{Stage 4: current and voltage stabilization period (normalized time $>10^{-5}$ )}

In this period, current, voltage, and heat flux start to stabilize toward steady state.

At the end of the calculation, the electron temperatures at the five locations range from 11000 to $38000 \mathrm{~K}$, which is around $1.0-3.3 \mathrm{eV}$. At the tip of the wire, the electron density is around $10^{-5}$ of the neutral particle density and the ion density is around $10^{-4}$ of the neutral particle density. The difference between the electron and ion densities can be attributed to the relative mobilities of the two particles. The mobility of the ions is much lower than the mobility of the electrons. Therefore, ions are more concentrated where they were first generated, i.e., the tip of the wire. Under the conditions of $R_{c}=100 \mathrm{k} \Omega, d=25.4 \mu \mathrm{m}$, and intereletrode gap $=10 \mathrm{~d}$, the steady-state voltage is around $400 \mathrm{~V}$, the current is around $26 \mathrm{~mA}$, and the total heat input to the wire is around $0.36 \mathrm{~W}$.

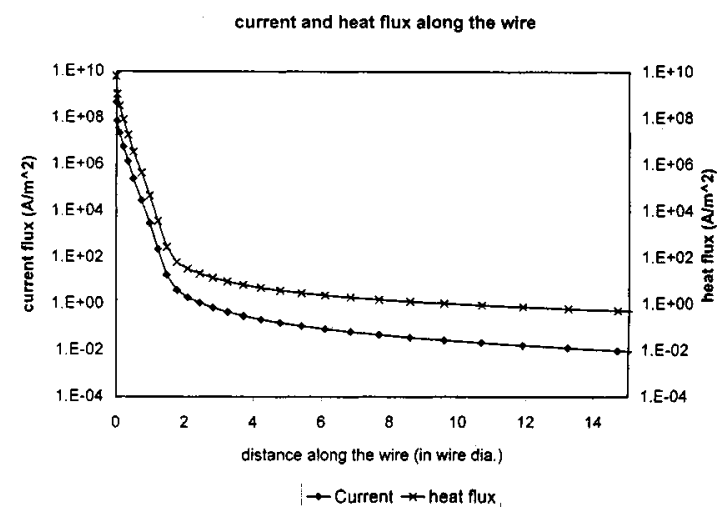

FIG. 12. Current flux and heat flux along the wire. $R_{c}=1 \times 10^{5} \Omega$, wire diameter $=25.4 \mu \mathrm{m}$, interelectrode gap $=0.254 \mathrm{~mm}$.

\section{B. Spatial distribution of charge particle densities, electron temperature, and current to the wire}

The electric field intensity is concentrated around the tip of the wire due to the very small diameter. Therefore, the electron temperature (Fig. 9) and charged particle densities (Figs. 10 and 11) will start to increase toward the wire tip. As the discharge develops, the densities start to rise at locations farther away from the tip. These can be observed from Figures 10 and 11. The peak electron density is about one decade $(10 \times)$ lower than the peak ion density due to the high mobility of the electrons and the electron absorption at the wire surface since the wire is the anode. However, at a certain distance away from the wire, the electron density is higher than the ion density. For example, for nondimensional time equal to $10^{-4}$, the electron density is slightly higher than the ion density in the middle of the gap.

The current and heat flux to the wire are plotted in Fig. 12. This figure indicates that the heat and current fluxes are concentrated at the tip of the wire and decrease rapidly away from the tip of the wire. The peak current density is around $5 \times 10^{8} \mathrm{~A} / \mathrm{m}^{2}$ and the peak heat flux is $6.4 \times 10^{9} \mathrm{~W} / \mathrm{m}^{2}$.

\section{Influence of ballast resistor, EFO gap, and wire diameter}

Three different values of the outer circuit resistance have been used in the calculation, $R_{c}=50,100$, and $200 \mathrm{k} \Omega$, respectively. The results for current and heat input to the wire are given in Table II.

For $R_{c}=0.5 \times 10^{5} \Omega$, the final (steady-state) current is 52 $\mathrm{mA}$. For $R_{c}=1 \times 10^{5} \Omega$, the final current is $26 \mathrm{~mA}$. For $R_{c}$ $=2 \times 10^{5} \Omega$, the final current is $13 \mathrm{~mA}$ and the potential of

TABLE II. Results for the discharge under different conditions.

\begin{tabular}{cccccc}
\hline \hline $\begin{array}{c}\text { Ballast } \\
\text { resistor }(\mathrm{k} \Omega)\end{array}$ & $\begin{array}{c}\text { Gap } \\
(\mu \mathrm{m})\end{array}$ & $\begin{array}{c}\text { Wire diameter } \\
(\mu \mathrm{m})\end{array}$ & $\begin{array}{c}\text { Current } \\
(\mathrm{mA})\end{array}$ & $\begin{array}{c}\text { Voltage } \\
(\mathrm{V})\end{array}$ & $\begin{array}{c}\text { Heat input } \\
(\mathrm{W})\end{array}$ \\
\hline 100 & 254 & 25.4 & -26.08 & -391.2 & 0.36 \\
200 & 254 & 25.4 & -13.04 & -391.0 & 0.18 \\
50 & 254 & 25.4 & -52.15 & -391.4 & 0.71 \\
100 & 203 & 25.4 & -26.08 & -391.6 & 0.36 \\
100 & 127 & 25.4 & -26.12 & -388.7 & 0.36 \\
100 & 254 & 31.8 & -26.06 & -394.5 & 0.33 \\
\hline \hline
\end{tabular}




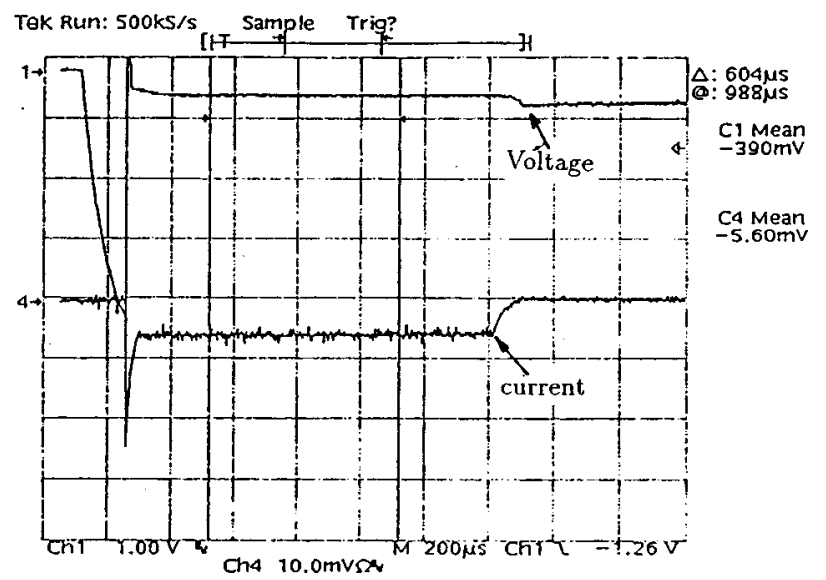

FIG. 13. Voltage and current waveforms for a wire to side wand discharge.

the wire is around $400 \mathrm{~V}$. This indicates that the ballast resistor can be used to effectively control the current flow in the discharge. The heat inputs to the wire are $0.71,0.36$, and $0.18 \mathrm{~W}$, respectively. Therefore, the relationship between the current and the heat flux appears linear.

Changing the gap also affects the outcome of the discharge. As the gap changes from ten to eight wire diameters, there is not much change in the current and heat flux. There is a slight increase in heat flux with a $0.127 \mathrm{~mm}$ gap. This is due to a small variation of the electric field intensity. In the case of two infinite parallel plates, the electric field intensity is inversely proportional to the distance between the two electrodes. However, in the wire to gap breakdown domain, the small diameter of the wire dominates the magnitude of the electric field intensity.

The wire diameter change has a bigger influence on the discharge than the gap length. From Table II we can see that increasing the wire diameter from 25.4 to $38 \mu \mathrm{m}$ results in more than a $7 \%$ decrease in heat flux. The peak initial electric field intensity decreases $17 \%$ from $7.1 \times 10^{7}$ to 5.9 $\times 10^{7} \mathrm{~V} / \mathrm{m}$ in this case.

\section{Comparison with experimental data}

Figure 13 shows the current and voltage waveforms for a similar discharge measured with a Tektronic digital oscilloscope. The experiment was set up with $25.4 \mu \mathrm{m}$ diam gold wire and a cathode to one side. The side wand was a metal rod with a semicircular tip. The vertical gap between the tip of the wire and the top of the wand was $0.25 \mathrm{~mm}$ and the horizontal gap was about $1 \mathrm{~mm}$. The wire was grounded through the wire clamp and a capacitor discharged negative high voltage to the cathode at the beginning of the breakdown. The ballast resistor was around $100 \mathrm{k} \Omega$ in the experiment.

The voltage was measured with an 1000:1 high-voltage probe. The current was measured with a dc current probe with the conversion of $50 \mathrm{~mA}: 10 \mathrm{mV}$. As the discharge reached steady state, the voltage was $390 \mathrm{~V}$ and the current was $28 \mathrm{~mA}$. This indicates a close match between the numerical calculation and the experimental data.
The formulation of the conservation equations used for the discharge is based on the linearized Boltzmann equations for a three-component plasma. There are several simplifications we made for this type of plasma. The accuracy of the calculation is also limited by the accuracy of the transport coefficients. Considering the complexity of the problem, the results of the simulation compare well with the experimental data. Most importantly, this simulation reveals the phenomena during the breakdown and the relationship between the heat transfer and the current levels. It is the first time results like this have been obtained.

\section{E. Comparison with previous numerical simulations}

Numerical simulations of a wire to plane discharge have been done by Ramakrishna, ${ }^{14}$ Donovan ${ }^{15}$ and $\mathrm{Jog}^{16}$ in their theses researches. Our results have many similarities with the previous results. First, all of the simulations predict that the highest charged particle densities occur at the tip of the wire where the electric field intensity is the highest. Second, we all showed that the ion density is higher than the electron density on the discharge axis (p. 72 in Ref. 14 and p. 21 in Ref. 16). Third, Jog's thesis showed that a rise in electron temperature precedes the increase in electron density and a subsequent decrease of the electron temperature as the ionization continues (p. 22 in Ref. 16). He also showed that the heat flux to the wire electrode is concentrated at the tip of the wire.

Quantitatively, the results of the work presented here and those of the previous theses differ significantly. The improvements in this paper that had not been done in any of the previous simulations are the following: (1) update of the voltage in the gap according to the current level at each time step; (2) use of a body fitted coordinate system for the solution domain, so the wire is simulated as a cylinder; (3) calculation of the electron temperature and formulation of the impact ionization term as a function of the electron temperature; and (4) a careful comparison between numerical and experimental results, obtaining close agreement by continuously improving the numerical code.

Some of the differences are results of the different solution domains and boundary conditions. Both Jog and Ramakrishna used $2500 \mathrm{~V}$ instead of $3000 \mathrm{~V}$, as in our calculation. The gap in their simulation is also twice as large $(0.5$ $\mathrm{mm}$ compared to $0.25 \mathrm{~mm}$ used here). However, the difference in the gap should not cause significant changes, as shown in Sec. V C. The major difference is the result of the improvements mentioned above. A few of the comparisons are given below.

For current flow to the wire, Ramakrishna ${ }^{14}$ calculated the nondimensional current for $2500 \mathrm{~V}$ between the wire and the wand to be around $10^{4}$, with the normalization current ( $\mathrm{p}$. 80 in his thesis) at $2.8 \times 10^{-9} \mathrm{~A}$, which results in a current less than $10^{-2} \mathrm{~mA}$. This is the current before breakdown, which is only one-thousandth of the steady-state current value obtained in this paper. His computation diverged as soon as current started to increase because there was no external ballast resistor to limit current in his simulation.

Jog's result ${ }^{16}$ for heat flux is also smaller than the results 
reported here. The heat flux from his calculation is around $4 \times 10^{7} \mathrm{~W} / \mathrm{m}^{2}$ (page 43 in Ref. 16). His result is only about one-hundredth of our calculation of $6.4 \times 10^{9} \mathrm{~W} / \mathrm{m}^{2}$, which is actually slightly smaller than the experimental result. The charged particle densities and electron temperature are also much smaller in his work. He obtained a peak electron temperature of less than $5000 \mathrm{~K}(0.4 \mathrm{eV})$, which is much smaller than the ionization energy, $14.87 \mathrm{eV}$. Here, the peak electron energy is close to the ionization energy and the start of the ionization process does not occur until this peak electron energy is reached.

\section{F. Effect of reduction of cross section}

Finally, we wanted to verify our intuition that reducing the cross section $\sigma_{T}$ would serve only to increase the time required for breakdown by lengthening each of the time periods described in Sec. V A. We performed the same computation with the cross section reduced by $10 \%$ with the following results. The time to break down was lengthened by $7 \%$ with no changes in the steady-state current or voltage (Fig. 7).

\section{CONCLUSIONS}

The formation of a wire-to-plane electric discharge in atmospheric pressure air has been numerically simulated from high-voltage prebreakdown through the ionization avalanche and current growth to stabilization and steady state. Two-dimensional transient solutions of the (mass and energy) conservation laws for ions and electrons with Gauss' law for the self-consistent electric field yield results in conformity with the corresponding experiments. Ionization and temperature growth profiles with time as well as their spatial variations are given. These results are improvements over prior ones in that the simulations reported here are closer to the actual physical occurrences.

\section{ACKNOWLEDGMENTS}

The authors especially appreciate the time and effort expended by the referee to make this a much better paper. The patience of the editor during a lengthy serious illness of one of the authors, followed by a catastrophic crash of our computer, resulting in more than three additional months of down time, is much appreciated. The authors gratefully acknowledge support for this work by the National Science Foundation, The Research Foundation of the University of Pennsylvania, and Kulicke and Soffa Industries, Inc.

${ }^{1}$ A. J. Davis, Proc. IEEE 133A, 217 (1986).

${ }^{2}$ J. P. Novak and R. Bartnikas, J. Appl. Phys. 62, 3605 (1987).

${ }^{3}$ S. T. Pai, J. Appl. Phys. 71, 5820 (1992).

${ }^{4}$ S. T. Pai and X. M. Guo, J. Appl. Phys. 71, 5826 (1992).

${ }^{5}$ M. Meyyappan and J. P. Kreskovsky, J. Appl. Phys. 68, 1506 (1990).

${ }^{6}$ D. B. Graves and K. F. Jensen, IEEE Trans. Plasma Sci. PS-14, 78 (1986).

${ }^{7}$ S. K. Park and J. Economou, J. Appl. Phys. 68, 3904 (1990).

${ }^{8}$ J. D. Cobine and E. E. Burger, J. Appl. Phys. 26, 895 (1955).

${ }^{9}$ K. Ramakrishna, I. M. Cohen, and P. S. Ayyaswamy, J. Appl. Phys. 65, 41 (1989).

${ }^{10}$ K. Ramakrishna, I. M. Cohen, and P. S. Ayyaswamy, J. Comput. Phys. 104, 173 (1993).

${ }^{11}$ K. G. Donovan and I. M. Cohen, J. Appl. Phys. 70, 4132 (1991).

${ }^{12}$ M. A. Jog, I. M. Cohen, and P. S. Ayyaswamy, Phys. Fluids B 3, 3532 (1991).

${ }^{13}$ W. Qin, I. M. Cohen, and P. S. Ayyaswamy, Trans. ASME, J. Electron. Packaging 116, 212 (1994).

${ }^{14}$ K. Ramakrishna, Ph.D. thesis, University of Pennsylvania, 1989.

${ }^{15}$ K. G. Donovan, M.S.E. thesis, University of Pennsylvania, 1989.

${ }^{16}$ M. A. Jog, Ph.D. thesis, University of Pennsylvania, 1993.

${ }^{17}$ J. F. Thompson, Z. U. A. Warsi, and C. W. Mastin, Numerical Grid Generation (Elsevier, New York, 1985).

${ }^{18}$ D. R. Wilkins and E. P. Gyftopolous, J. Appl. Phys. 37, 3533 (1966).

${ }^{19}$ W. P. Allis, in Handbuch der Physik, XXI, edited by S. Flügge (SpringerVerlag, Berlin, 1956), pp. 384-444.

${ }^{20}$ S. C. Brown, Basic Data of Plasma Physics, 2nd ed. (M.I.T. Press, Cambridge, 1967), pp. 182-186.

${ }^{21}$ A. von Engel, Ionized Gases, 2nd ed. (Oxford U. P., London, 1965), pp. $179-182$.

${ }^{22}$ P. M. Chung, Phys. Fluids 12, 1623 (1969).

${ }^{23}$ C. A. Fletcher, Computational Techniques for Fluid Dynamics (SpringerVerlag, New York, 1991), pp. 252-253.

${ }^{24}$ S. V. Patankar, Numerical Heat Transfer and Fluid Flow (Hemisphere, Washington, DC, 1980), pp. 90-100. 